\title{
A Hybrid Simulation Model for ICT-based Wide Area Damping Control of Power System
}

\author{
Xingyu Shi ${ }^{\mathrm{a}}$, Zhisheng $\mathrm{Xu}^{\mathrm{b}}$, Yong $\mathrm{Li}^{\mathrm{a}^{*}}$, Yijia Cao ${ }^{\mathrm{a}}$, Chen Zhang ${ }^{\mathrm{c}}$, Min Wen ${ }^{\mathrm{b}}$ \\ ${ }^{a}$ College of Electrical and Information Engineering, Hunan University, Changsha and 410082, China \\ ${ }^{\mathrm{b}}$ State Power Economic Research Institute of Hunan Province, Changsha 410004, China \\ ${ }^{\mathrm{c}}$ Shanghai Data Converging Co., Ltd, Shanghai and 200030, China \\ *Corresponding Author: yongli@hnu.edu.cn
}

\begin{abstract}
In this paper, a hybrid simulation model is proposed to evaluate the performance of wide area damping control (WADC) of power system, which is used to damp out the low-frequency oscillation (LFO). This model considers the information and communication technology (ICT) used for implementation of WADC. The UDP/IP protocol is used to transmit operation and control data between power system and WADC controller. A flexible ac transmission system (FACTS) based WADC controller is designed by using phase compensation method and implemented by C\#. In this way, a wide area closed-loop control system is formed. Finally, a IEEE benchmark system is used in case study, equipped with a shunt-type FACTS device. And a line-to-ground fault in such system is used to validate the established hybrid simulation model as well as the WADC performance.
\end{abstract}

Keywords: WADC, ICT, LFO, Hybrid simulation.

\section{Introduction}

In large-scale interconnected power systems, long distance bulk power transfer could cause many modes of oscillation as a consequence of interactions of its components. Those oscillations appear in the transmission line between two or more generators and endanger the power system stability.

Low-frequency oscillation (LFO) includes local mode and inter-area oscillations whose frequency of oscillation are in the range of $1-2 \mathrm{~Hz}$ and $0.1-1 \mathrm{~Hz}$, respectively ${ }^{(1)}$. If power systems lack sufficient and effective damping, LFO will cause some problems such as lowering power transfer capacity of the transmission lines and negatively affecting bus voltage, line current and generator speed.

To ensure secure and reliable operations, the large-scale interconnected power systems require wide area observation and control. To meet these requirements, many wide area control methodologies have been established. Since the synchronized phasor measurement unit (PMU) based on the global positioning system (GPS) was invented in the early 1990s, one of the most well accepted approaches called wide area monitoring/measurement system (WAMS) based on wide area synchrophasor measurement technology was introduced to realize the collection and dynamic monitoring of real-time data such as voltage, current, angle, frequency from different nodes, analysis and control for stable and efficient operation of the global power system. At present, WAMS based LFO monitoring has been already applied in the world ${ }^{(2)}$.

The wide area damping control (WADC) is regarded as a functional extension of WAMS. Utilizing the information and communication technology (ICT) infrastructure together with suitable algorithms, the WADC achieves real-time wide area measurement, real-time data exchange and fast coordinated control and actions. At present, in order to obtain better damping control, designing and implementing a better WADC control system to improve the damping of power system low-frequency inter-area oscillations is significant. WADC systems have many benefits, they not only improve the damping performance and the stability of power systems, but also provide better analysis and control of power systems in contrast to conventional local control.

The main works of the paper are as follows: (a) Establish a hybrid simulation model considering ICT to represent power system and WADC. (b) Design a WADC controller, which are considered for large interconnected 
systems, and propose a systematic procedure to design WADC systems and show its performance of damping out LFO. (c) A case study is used to validate the established hybrid simulation model as well as the designed WADC.

The paper is structured as follows: Section 2 presents the architecture of hybrid simulation model. The design and implementation of WADC controller are proposed in Section 3. To test the damping performance of WADC, real-time hybrid simulation and verification are carried out in Section 4. Conclusions are given in Section 5.

\section{Architecture of Hybrid Simulation Model}

In this section, a hybrid simulation is presented, consisting of physical and cyber systems, as illustrated in Fig. 1. In the hybrid simulation model, the physical system is developed to account for relevant components interacting in the operation of power system. The cyber system, utilizing ICT, is set up to implement and evaluate the control performance of enhanced WAMS center.

During the simulation, the power system sends the actual signal data (i.e., rotor speeds from the remote generators) to enhanced WAMS center. Then its control comments are calculated by using the designed algorithm. Finally the supplementary control output signals are sent to the controllable device.

The aim of adopting two computers is to utilize the actual Ethernet based communication network between them and to analyze the influence of communication network on control performance of enhanced WAMS center, which is the future work.

The simulation and validation are carried out in two separate computers of different areas. The IEEE benchmark test system with a FACTS controller, which is built in MALAB, runs on Computer 1 in one area. While the enhanced WAMS center, which is implemented using C\#, runs on Computer 2 in another area. The two computers communicate with each other based on the Ethernet network.

\subsection{Power System Model}

The IEEE benchmark test system, shown in Fig. 2, consists of two identical areas connected through three-phase transmission $\operatorname{line}^{(3)}$. Each area includes a generating unit and a shunt FACTS device is placed in the middle of the interconnected line to improve the transmission capability. The communication blocks are used to receive supplementary control signals and send operation data to enhanced WAMS center via Ethernet. The dominant inter-area oscillation mode is determined by the network structure and the dynamic behavior of both generation sides G1 and G2.

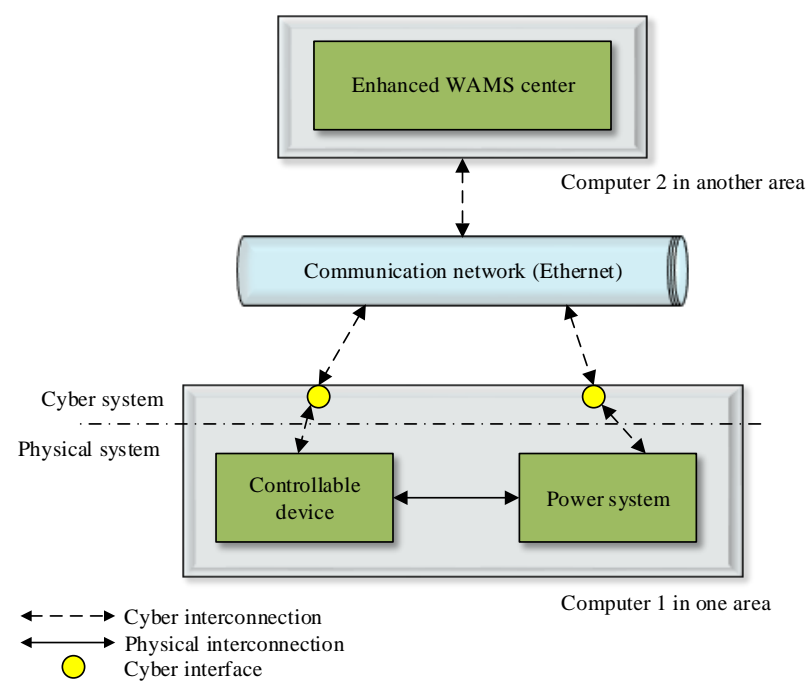

Fig. 1. Framework of the Hybrid Simulation Model.

\subsection{Communication protocol}

In general, the transmission control protocol (TCP) and user datagram protocol (UDP) are the two most famous transport protocols, for data transmitting between network applications. Together with internet protocol (IP), they are referred to as TCP/IP and UDP/IP. Both protocols can be used in different fields ${ }^{(4)}$. However, due to acknowledgment and handshaking, some overhead information are added to the TCP header section, which makes it slow in data transmission processing. In this paper, the data transmission is based on the UDP/IP to perform a real-time closed-loop control via a utility communication network.

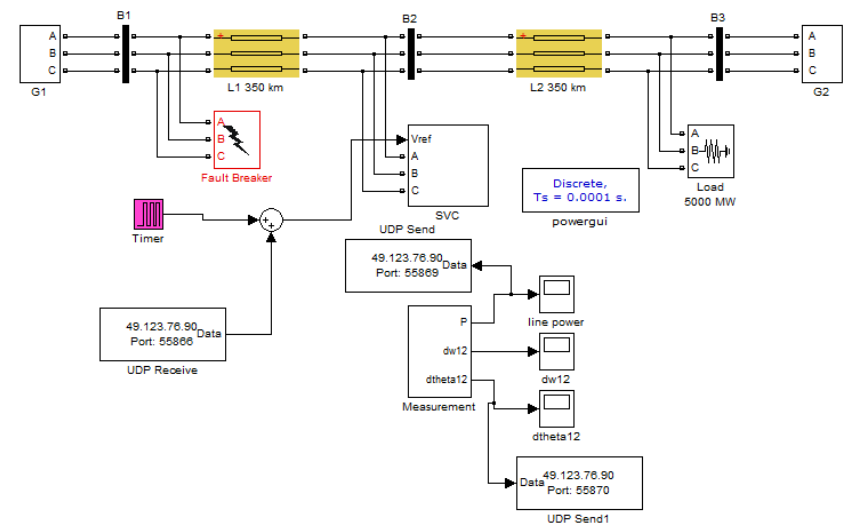

Fig. 2. A Typical Interconnected System with FACTS Device. 


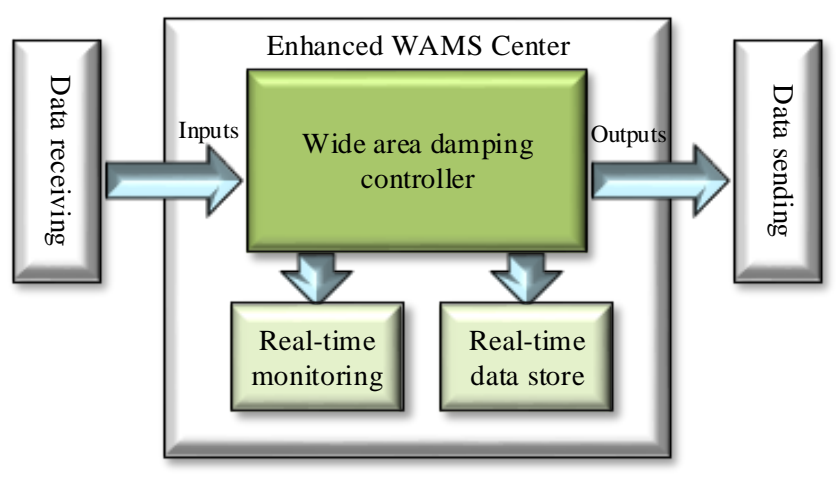

Fig. 3. Enhanced WAMS Center.

\subsection{Enhanced WAMS Center}

The WAMS center and the WADC controller form an enhanced WAMS center with the wide area stability control ability to achieve the acceptable damping performance. Such center is implemented by C\# language. The enhanced WAMS center, as shown in Fig. 3, mainly has three parts. It includes a WADC part, a power system stability monitoring view part and a real-time data storage part. The design and implement of WADC controller will be discussed in the next section.

\section{Design of WADC Controller}

Linear analysis of the power system model is performed. The right eigenvectors (mode shape) with respect to the rotor angle and speed states of the generators indicate an inter-area oscillation mode having the eigenvalue, $\lambda=-0.026 \pm j 4.24$, frequency, $f=0.67$, damping ratio $\zeta=0.006$. This mode corresponds to the inter-area oscillation of G1 against G2. The damping ratio of 0.006 should be improved to enhance system stability.

\subsection{Design Principle}

The WADC design procedure is based on the residue approach $^{(5)}$. Fig. 4 shows the closed-loop system with a WADC controller.

The transfer function $G(s)$ can be expanded in partial

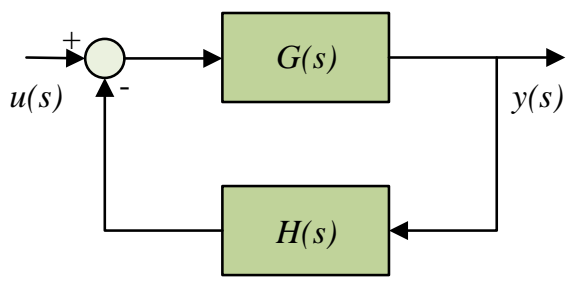

Fig. 4. Closed-loop System with a WADC Controller.

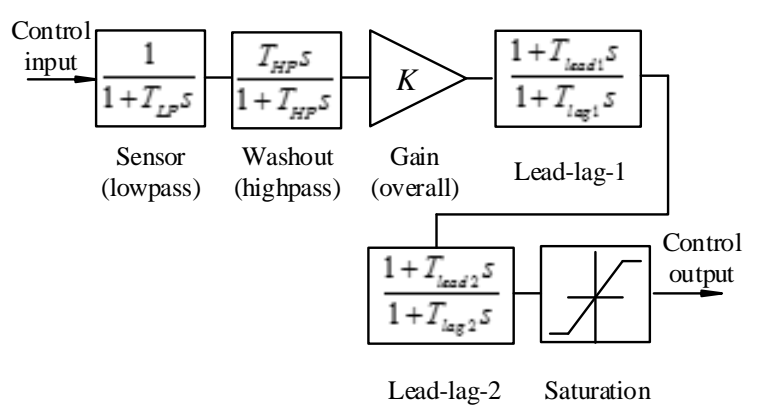

Fig. 5. Structure of the FACTS controller. fractions with residue $R_{i}$ and eigenvalue $\lambda_{i}$, that is ${ }^{(5)}$,

$$
G(\mathrm{~s})=\frac{R_{1}}{s-\lambda_{1}}+\frac{R_{2}}{s-\lambda_{2}}+\ldots+\frac{R_{n}}{s-\lambda_{n}}=\sum_{i=1}^{n} \frac{R_{i}}{s-\lambda_{i}}
$$

where $R i$ is the residue of the transfer function $G(s)$ associated with the eigenvalues $\lambda_{i}$ at the mode- $i$.

The WADC controller consists of an amplification block, a high-pass filter, a low-pass filter and $m_{c}$ stages of lead-lag blocks, as shown in Fig. 5. According to Fig.5, the transfer function $H(s)$ of the FACTS WADC controller can be expressed as:

$$
H(\mathrm{~s})=\mathrm{K}\left(\frac{1}{1+T_{L P} s}\right)\left(\frac{T_{H P} s}{1+T_{H P} s}\right)\left(\frac{1+T_{\text {lead }} s}{1+T_{\text {lag }} s}\right)^{m_{c}}=K H_{1}(\mathrm{~s})
$$

where $K$ is a control gain; $T_{L P}$ and $T_{H P}$ are the time constants of the low-pass filter and the high-pass filter respectively, $T_{\text {lead }}$ and $T_{\text {lag }}$ are the lead and lag time constant, respectively. Generally, two lead-lag blocks are used. $H_{1}(s)$ is the phase compensation transfer function.

For the closed-loop system with the feedback transfer function $H(s)$, the eigenvalue sensitivity is shown in (3), which gives the relationship between the sensitivity of eigenvalue to feedback loop gain and the open loop residue associated with the same eigenvalue.

$$
\frac{\partial \lambda_{i}}{\partial K}=R_{i} \frac{\partial H\left(\lambda_{i}\right)}{\partial K}=R_{i} \frac{\partial K H_{1}\left(\lambda_{i}\right)}{\partial K}=R_{i} H\left(\lambda_{i}\right)
$$

Therefore assuming the gain $K$ is small enough and adding the feedback to the system, it will cause a change in eigenvalue in the initial operating point ${ }^{(5)}$.

$$
\Delta \lambda_{i}=R_{i} K H\left(\lambda_{i}\right)
$$

From (4), it can be observed that when the feedback control $H(s)$ is applied, the eigenvalues of the initial system will be changed. The objective of the FACTS damping controller is to improve the damping ratio of the selected oscillation model $i$, and $\Delta \lambda_{i}$ must be a real negative value to move the real part of the eigenvalue to the left half 


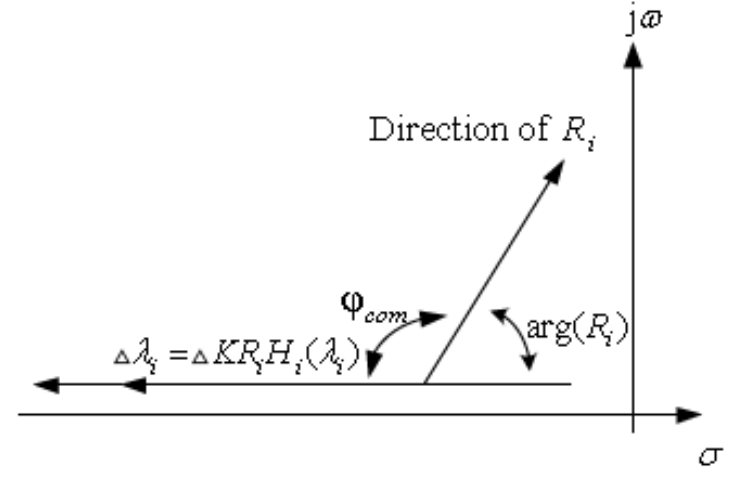

Fig. 6. Concept of Phase Compensation.

negative complex plane without changing the frequency.

Fig. 6 shows the movement of the eigenvalue of the system with a FACTS based WADC controller $^{(5)}$. The compensation angle is used to move the eigenvalue direct to the negative axis. In this figure, the phase angle $\varphi_{\text {com }}$ shows the compensation angle, which is needed to move the eigenvalue directly to the left parallel with the real axis. The phase angle $\varphi_{\text {com }}$ can be achieved via the lead-lag function. The parameters of the lead and lag time constants, $T_{\text {lead }}$ and $T_{\text {lag }}$, can be determined as follows ${ }^{(5)}$ :

$$
\begin{aligned}
& \varphi_{\text {com }}=180^{\circ}-\arg \left(\mathrm{R}_{i}\right), \alpha=\frac{T_{\text {lead }}}{T_{\text {lag }}}=\frac{1-\sin \frac{\varphi_{\text {com }}}{m_{c}}}{1+\sin \frac{\varphi_{\text {com }}}{m_{c}}} \\
& T_{\text {lag }}=\frac{1}{\omega_{n} \sqrt{\alpha}}, T_{\text {lead }}=\alpha T_{\text {lag }}
\end{aligned}
$$

where $\varphi_{\text {com }}$ is the phase angle; $\arg \left(R_{i}\right)$ is the phase angle of the residue $R_{i}, \omega_{n}$ is the frequency of the dominant oscillation mode. The parameter $m_{c}$ is the number of the lead-lag blocks. Usually two blocks are sufficient to compensate the input signal, while the angle compensated by each block should not exceed $60^{\circ}{ }^{(5)}$.

The WADC controller gain $K$ has to be optimized for the damping of all modes of oscillation because it is proportional for the feedback of the closed-loop system and the controller gain. The damping effectiveness of the WADC controller is influenced by $K$. As shown in Fig.6, the eigenvalue $\lambda_{i}$ from the original operating point with the increase of $\mathrm{K}$ will move to the negative axis. Therefore the $K$ can be computed as a function of the desired eigenvalue location according to the following equation ${ }^{(5)}$ :

$$
K=\left|\frac{\Delta \lambda_{i}}{R_{i} H_{1}\left(\lambda_{i}\right)}\right|=\left|\frac{\lambda_{i, \text { des }}-\lambda_{i}}{R_{i} H_{1}\left(\lambda_{i}\right)}\right|
$$

where $\lambda_{i, d e s}$ is the desired eigenvalue. In addition, the value of $K$ can be determined using the root-locus method ${ }^{(6)}$.
Table 1. Controller Parameters.

\begin{tabular}{|c|c|}
\hline Items & Values \\
\hline Time constant of low pass filter $T_{L P}$ & 0.001 \\
\hline Time constant of low pass filter $T_{H P}$ & 1 \\
\hline Gain of controller $K$ & 0.01 \\
\hline $1^{\text {st }}$ lead time constant $T_{\text {leadl }}$ & 0.09 \\
\hline $1^{\text {st }}$ lag time constant $T_{\text {lagl }}$ & 0.6181 \\
\hline $2^{\text {nd }}$ lead time constant $T_{\text {lead } 2}$ & 0.09 \\
\hline $2^{\text {nd }}$ lag time constant $T_{\text {lag } 2}$ & 0.6181 \\
\hline
\end{tabular}

The parameters of the WADC controller are indicated in Table 1. The values of $T_{L P}$ and $T_{H P}$ are set from the empirical values.

Because the WADC controller is only a supplementary control module of the shunt-type FACTS device, the control output of the WADC controller is limited in range of $-0.2 \mathrm{pu}$ to $0.2 \mathrm{pu}$ in order to avoid influence on the local control of FACTS devices. Therefore a saturation block must be added to set a limitation for the output signals. In fact, the control output signal $\Delta U$ is a supplementary control signal to the controllable device. Thus it forms a wide area closed-loop stability control while the control algorithm works.

\subsection{Discrete-time Model of Designed Controller}

In order to implement the designed controller by programing, the continuous transfer function must be converted to a discrete transfer function. That means the continuous transfer function must be converted from s-domain to z-domain by using Z-transform. Tustin transformation, also called bilinear transformation, is used to convert continuous transfer functions to discrete transfer functions $^{(7)}$.

After discretizing transfer functions and comparing it with continuous transfer functions, a transformation relation can be found between s-plane and z-plane, namely:

$$
s=\frac{T_{s}}{2} \frac{z-1}{z+1}
$$

where $T_{s}$ is sample time.

As mentioned before, the block diagram of the structure of the FACTS controller involves a five-stage transfer function. By applying the transformation relation (7), each transfer function can be expressed as follows:

(a) The low pass filter in discrete time, $H_{L P}(z)$, is:

$$
H_{L P}(\mathrm{z})=\frac{\frac{T_{s}}{T_{s}+2 T_{L P}} z+\frac{T_{s}}{T_{s}+2 T_{L P}}}{z+\frac{T_{s}-2 T_{L P}}{T_{s}+2 T_{L P}}}
$$


(b) The high pass filter in discrete time, $H_{H P}(z)$, is:

$$
H_{H P}(\mathrm{z})=\frac{T_{H P} \frac{2}{T_{s}} \frac{z-1}{z+1}}{1+T_{H P} \frac{2}{T_{s}} \frac{z-1}{z+1}}
$$

(c) The lead-lag block in discreet time is:

$$
H_{L-L}(\mathrm{z})=\frac{\frac{T_{s}+2 T_{\text {lead }}}{T_{s}+2 T_{\text {lag }}} z+\frac{T_{s}-2 T_{\text {lead }}}{T_{s}+2 T_{\text {lag }}}}{z+\frac{T_{s}-2 T_{\text {lead }}}{T_{s}+2 T_{\text {lag }}}}
$$

According to (8)-(10), the Transfer function $H(z)$ is expressed as below, where $Y(z)$ and $U(z)$ represent the input and output signals of $H(z)$, respectively:

$$
\begin{aligned}
& H(\mathrm{z})=\frac{Y(\mathrm{z})}{U(\mathrm{z})}=\frac{K_{n u m 1} z+K_{n u m 2}}{z+K_{d e n}} \\
& z Y(\mathrm{z})=\mathrm{K}_{d e n} Y(\mathrm{z})=\mathrm{K}_{n u m 1} z U(\mathrm{z})+\mathrm{K}_{\text {num } 2} U(\mathrm{z}) \\
& \mathrm{Y}(\mathrm{n})=\mathrm{K}_{d e n} Y(\mathrm{n}-1)=\mathrm{K}_{n u m 1} U(\mathrm{n})+K_{n u m 2} U(\mathrm{n}-1) \\
& Y(\mathrm{n})=\mathrm{K}_{\text {num } 1} U(\mathrm{n})+\mathrm{K}_{\text {num } 2} U(\mathrm{n}-1)-\mathrm{K}_{d e n} Y(\mathrm{n}-1)
\end{aligned}
$$

The Z-transform above can be implemented via using Mircosoft C\# programming language.

\subsection{Controller Implementation}

According to the last equation in (11), at least three values are needed in each iteration step. Here the actual input value, the last input value and last output value are necessary. The last input value must be stored for the next calculation.

The calculation process of output signals of the FACTS WADC controller consists of five steps. The discrete sequence is first transmitted into the low pass model and then goes through the high pass model, the gain and two lead-lag models. After saturation treatments, the controlled sequence is used as the control output of the WADC controller and is transmitted to the controllable device in the power system model.

For the C\# implementation, the actual input value and the last input value for the WADC controller are stored in array $x$ [1] and x [0] respectively. As mentioned before, the output value of the low pass filter is the input of the high pass filter. Therefore, after the data is transmitted into the low pass model, the new input value and the new last input value are stored in y [1] and y [0], respectively. Then it runs through the rest blocks shown in Fig.7. Finally w [1] stores the actual output value of the second lead-lag model. The resulting output value is the control output of the WADC controller.

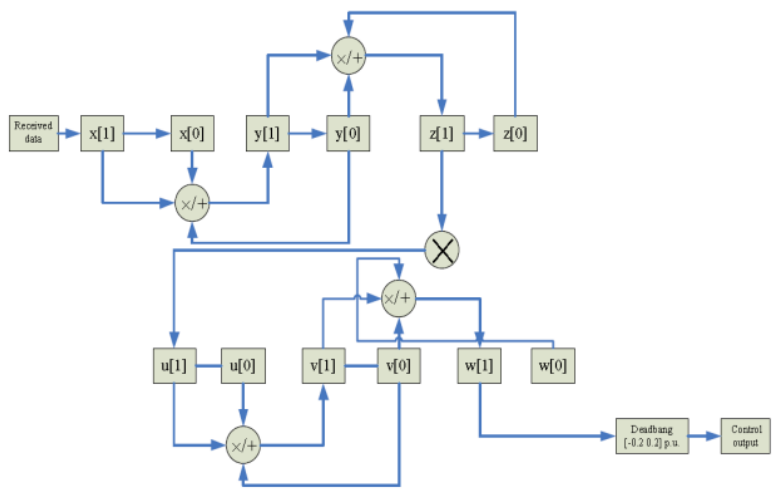

Fig.7. Flowchart of control algorithm implemented by C\#.

\section{Case Study}

To test the damping performance of the inter-area oscillation in the interconnected power system, real-time hybrid simulation and verification are carried out between two computers in the MATLAB/Simulink and Microsoft Visual Studio environment, respectively. A line-to-ground fault (big disturbance) near Bus-1 that happens at $1.0 \mathrm{~s}$ and is cleared at $1.1 \mathrm{~s}$, is used to illustrate the performance of the designed WADC on the stability of the interconnected system.

The IEEE benchmark system equipped with a shunt-type FACTS device is established using MATLAB/SimPowerSystems on Computer 1. The Computer 1 is assigned with a static IP address, and the WADC controller, whose parameters are shown in Table 1, provides a supplementary control to the equipped shunt-type FACTS device on computer 2, which also has a static IP address. The sample time in the simulation is set as $T_{s}=0.0001 \mathrm{~s}$.

Fig. 8 shows the control input response of the WADC controller using designed control algorithms. Compared with the open-loop simulation result before, the inter-area oscillation can be completely damped within about $12 \mathrm{~s}$. It means that the inter-area oscillation is effectively damped

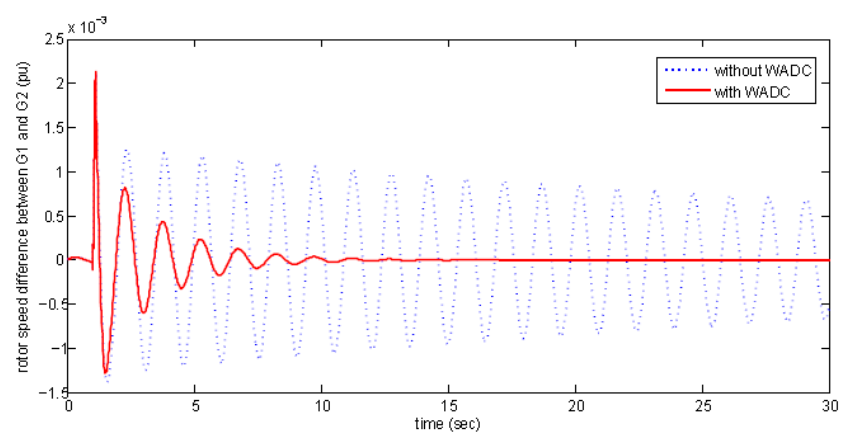

Fig. 8. Dynamic response of the rotor speed difference. 


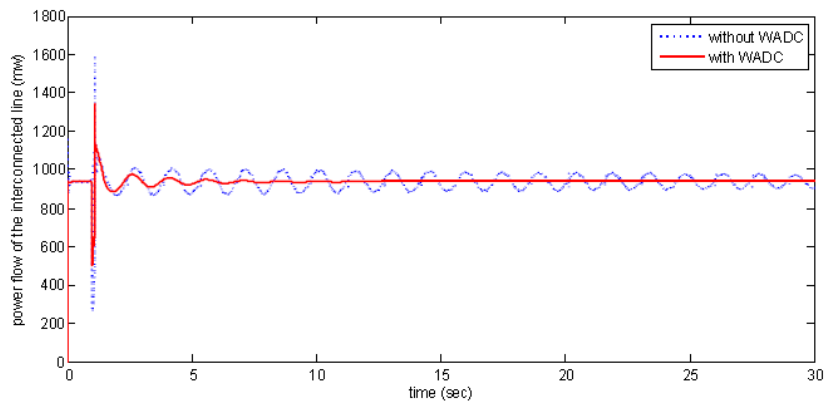

Fig.9. Dynamic response of power flow on the interconnected line.

with wide area closed-loop control.

Fig.9 illustrates that the power oscillation in the interconnected line is damped in about $10 \mathrm{~s}$, which means the designed WADC controller is able to respond to the system instability and provide effective damping to reduce the inter-area oscillation.

The simulation results indicate that the established hybrid simulation model and designed WADC is able to effectively dampen the inter-area oscillations in the interconnected power system. It can be regarded as a functional extension of the current WAMS. In the whole data transmission process, the transmission delay is not considered.

\section{Conclusion}

In this paper, a hybrid simulation model is proposed to evaluate the performance of WADC of power system. In the model, ICT with UDP/IP protocol is used to transmit the wide area signals for WADC implementation. Using the phase compensation method, a FACTS based WADC controller is designed in s-domain and in order to implement the designed controller by $\mathrm{C \#}$ programing, such controller is converted to $\mathrm{z}$-domain by Tustin transformation. Finally, the case study shows the good performance of the established hybrid simulation model and designed WADC.

\section{Acknowledgment}

This work was supported by the Key Program of the National Natural Science Foundation of China under Grant 61233008 and the National Science and Technology Support Program of China under Grant 2013BAA01B01.

\section{References}

(1) P.Kundur: "Power System Stability and Control", McGraw-Hill: New York, 1994

(2) Xiaorong Xie, Yaozhong Xin, Jinyu Xiao, Jingtao Wu and Yingdao Han: "WAMS Applications in Chinese Power Systems", Power and Energy Magazine, IEEE, pp. 54-63, 2006

(3) D. Jovcic and G.N. Pillai: "Analytical Modeling of TCSC Dynamics", Power Delivery, IEEE Transactions, pp. 1097-1104, 2005

(4) W. Richard Stevens and Gary R. Wright: "TCP/IP Illustrated", Addison-Wesley Longman, Amsterdam, Vol. 2, 1995

(5) Ning Yang, Qinghua Liu and James D.McCalley: "TCSC Controller Design for Damping Inter-area Oscillations", IEEE Transaction on Power System, Vol. 13, No. 4, pp. 1304-1310, 1998

(6) H.M.Ayres, I.Kopcak, M.S.Castro, F.Milano and V.F.da Costa: "A Didactic Procedure for Designing Power Oscillation Dampers of FACTS Devices", Simulation Modelling Practice and Theory, pp. 869-909, 2010

(7) V. Kamaraju and R.L. Ralasimham: "Linear Systems-Analysis and Applications", I. K. International Publishing House Pvt. Ltd, 2009 